\title{
Evaluation of Tibia Bone Healing by Infrared Thermography: A Case Study
}

\author{
Wally auf der Strasse (D) \\ Daniel Prado Campos (D) \\ Celso Júnio Aguiar Mendonça (iD) \\ Jamil Faissal Soni iD ${ }^{2}$ \\ Joaquim Mendes iD ${ }^{3}$ \\ Percy Nohama (iD) ${ }^{1,2}$ \\ 'Universidade Tecnológica Federal do \\ Paraná, Curitiba, Paraná, Brasil; ${ }^{2}$ Pontifícia \\ Universidade Católica do Paraná, \\ Curitiba, Paraná, Brasil; ${ }^{3}$ INEGI, \\ Faculdade de Engenharia, Universidade \\ do Porto, Porto, Portugal
}

Correspondence: Wally auf der Strasse Rua Omílio Monteiro Soares, I360, Fanny, Curitiba, Paraná, CEP 8I.030-00I, Brasil Tel +55 4l 99974-6692

Email wallystrasse@hotmail.com
Background: Thermal imaging has been used as a clinical follow-up technique in several medical specialties.

Purpose: The aim of this study was to investigate the feasibility of using medical thermography in the diagnosis and follow-up assessment of a severe orthopedic trauma that requires the use of an external circular fixator.

Patients and Methods: Twenty clinical follow-ups of thermal imaging correlated with $\mathrm{X}$-ray images were performed in a male volunteer, diagnosed with bone nonunion, during 11 months of treatment, in the hospital trauma and reconstruction department. Data were acquired in the regions of interest of the proximal tibia, diaphysis and distal, with a Flir T530 medical grade infrared camera from Flir Systems ${ }^{\circledR}$, and the data processed by the Matlab $^{\circledR} 2019$ custom made software.

Results: Statistical analysis was performed by Wilcoxon signed-rank test. The results showed a median temperature of $22.2^{\circ} \mathrm{C}$, and thus some periods of interruption in the healing process between the third and twentieth clinical follow-up, and a significant increase of the temperature to $34.6^{\circ} \mathrm{C}$ synchronous with a diagnosis of bone infection by the eleventh clinical follow-up. The thermal images acquired during the 20 clinical follow-ups allow a correlation with the data from the X-ray exams and also with the contralateral limb of the evaluated patient, showing thermal alterations greater than $0.3^{\circ} \mathrm{C}$, which are significant of physiological abnormality.

Conclusion: The thermography exam can be a useful tool for applying on the follow-up of patients after trauma or bone fracture. The results showed important physiological data related to the vascularization necessary for bone repairing, being therefore a good indicator of the healing process. In addition, as infrared thermography does not use ionizing radiation, it can be used countlessly, in complement to the traditional X-ray exams that focus on anatomical data analysis.

Keywords: infrared medical thermography, nonunion, bone healing, Ilizarov method

\section{Introduction}

Nonunion bone, is a bone-articular pathology caused by unstable bone consolidation after a major injury of the bone tissue and its consequent osteogenesis production. ${ }^{1}$ This pathology can arise during the healing process of a bone fracture and consists of the absence of new bone formation during the period of bone healing, which can be aggravated by segmental bone loss, infections, type of fracture, deformity, or failure of internal fixation.

The lack of progression of the healing process, commonly diagnosed based on radiographic evidence, is characterized by one of the following factors: sclerosis at the end of the fracture, presence of hiatus, absent or hypertrophic callus, and 
persistence or widening of the fracture line. ${ }^{2}$ The Ilizarov method $^{3,4}$ is considered an excellent technique for treating segmental fractures of severe fractures in long bones (femur, tibia, and humerus), when accompanied by large bone losses or bone infection (osteomyelitis) that progress to non-bone consolidation (nonunion). ${ }^{5,6}$ The external fixator is mechanical linked to the bone, therefore, perforating the skin in several locations which, together with the biofilm that will be formed in these parts will contribute to the contamination and infection of the area, slowing down the treatment. To minimize this problem, antibiotics are usually administered to the patients for long periods. ${ }^{7}$

Surgical techniques of radical debridement of bone ends are widely used in the departments of trauma and bone reconstruction; being the most common cases fractures in tibia bones caused by serious car accidents due to high energy trauma, portraying a difficult problem for orthopedists throughout the world. ${ }^{8}$ The external circular fixation Ilizarov method (ECFIM) uses thin wires and/or pins inserted percutaneously fixed and tensioned to provide a strong supporting structure, enabling internal bone fixation in order to promote bone healing. ${ }^{9}$

In bone fractures that do not present complications, the average healing time is six months, while in high-energy bone trauma, depending on the severity of the fracture, treatment is surgical and may require the use of a circular external fixator extending the treatment time to eight months or more. However, in fractures that present significant segmental bone loss, surgical treatment includes the processes of lengthening bone and bone transport, thus, the time of treatment and medical follow-up can last for several years, depending on the amount of bone lost. The individual healing response presented by each patient, his nutritional state, and other associated diseases can also be determinant factors for the length of the clinical treatment.

The renowned Ilizarov's technique, when combined with the osteotomy procedure, ${ }^{10}$ promotes the local blood supply and consequently bone healing, but also increases the bone infections due to the pin/cable links. Meanwhile, for patients who present local soft tissue loss in the injured area, bone transport can provide soft tissue repair, as the bone is pulled to an appropriate length for each patient. ${ }^{11}$ Within a few days, the traction force applied during bone transport stimulates soft tissue regeneration together with blood vessels and peripheral nerves around the area, contributing to repairing the surrounding tissues. ${ }^{12}$ The healing process, bone formation and remodeling, is directly related to metabolic and vascular activity at the fracture site, creating warmer and cooler areas, according to skin perfusion at the fracture site, bone transport and bone elongation processes.

Successful bone repair relies heavily on the formation of new blood vessels to ensure the supply of nutrients and oxygen, as well as the removal of metabolites. Bone morphogenetic proteins (BMPs) and vascular endothelial growth factor (VEGF) are involved in cell differentiation and bone vascularization in order to develop viable bone tissue. ${ }^{13,14}$ A sufficient vascular network plays an important role in the repair of bone defects. When there is no formation of new blood vessels, there is a decrease in temperature at the fracture site, which is a sign of interruption of the healing process. According to the International Consensus and International Guidelines for Medical Thermography, temperature measured by thermographic examination in bone fractures below $23^{\circ} \mathrm{C}$ indicates interruption of bone healing. Thus, the assessment of the temperature in the period corresponding to the bone consolidation phase can support the physician in clinical follow-up decisions. ${ }^{15,16}$ Due to the anatomical structure of the lower limbs and according to the severity of the bone trauma, previous surgeries and the patient's treatment time, blood perfusion (biological supply) may be relatively poor in nonunion in the tibia diaphysis, with an emphasis on lesions in the distal third. Thus, disturbed healing and the occurrence of failures in the bone remodeling process until the bone union takes place are very common in this region of the body when compared to other sites. ${ }^{9}$

Together with the treatment with the ECFIM, when observing the temperature differences in the affected regions and between the insertion rings of the external fixator, one can denote the interruption of the bone remodeling process and establish a thermal threshold in the development of the infection. By analyzing the change in the blood perfusion, the diagnosis through medical thermography examination allows the correlation with radiographic images, in the evaluation of the bone remodeling stage at the fracture site. This allows the orthopedic surgeons to initiate gradual loading protocols to the affected limb, that is, placing the foot on the ground to gradually support the gait, with the intention of providing biological stimulation to bone healing. ${ }^{17,18}$

Internal thermal changes during bone healing are reflected in adjacent tissues and related to changes in blood flow and/or infection of the trauma region which eventually manifested as changes at skin temperature level. Thermography is a diagnostic technology that can 
detect epidermis thermal variations, so it can only be applied to bone fractures or musculoskeletal injuries in superficial bones, like the tibial.

Medical infrared thermography (IRT) provides twodimensional temperature images without the harmful effects of ionizing X-ray radiation. ${ }^{19}$ IRT is a noninvasive, noncontact, nonradioactive and low-cost diagnostic technology, allowing the acquisition of sequential images, capable of analyzing thermal variation related to physiological changes at the skin level. ${ }^{19}$ Another practical advantage is that the infrared camera is portable, and thus can be brought to the patient, directly in the clinical environment, without the need to take the patient to an imaging department, being therefore easier to use. However, it is also important to highlight the difficulties of making a thermal evaluation if there is no proper room with a controlled environment and low interference from metallic medical tools in the vicinity of the patient (which can increase the reflected radiation and thus measurement temperature errors). Anatomical data offered by radiographic images together with the physiological thermal information provided by an infrared camera allow a more complete diagnosis, to tune the treatment protocols and support medical decisions, like new surgeries.

A good vascularization, with an ample blood supply for bone healing is recognized as an important factor, as ischemic and hypoxic environments do not favor bone repair. $^{20}$ The applicability of infrared imaging in bone lesions has been described by De Sallis et $\mathrm{al}^{21}$ in their investigation in children diagnosed with osteogenesis imperfect (brittle bones), also use thermography to assess changes in skin temperature to aid diagnosis and screening vertebral fractures. Likewise, Reed et al $^{17}$ investigated the applicability of IRT in pediatrics, as a screening tool in the diagnosis of fractures and musculoskeletal wrist injuries, as these traumas are very common in children. However, the fracture line, or ligament and joints injuries, are not always visible in the first radiographic image making a second exam necessary. The aim of the authors was to investigate the use of IRT in the verification of thermal changes in the skin in the immediate inflammatory response to trauma, or diagnosis of a bone fracture. For this study, 105 children were evaluated to differentiate the diagnoses of wrist torsion and fractures. In the thermogram analyses, thermal cutoff values were set to establish the temperature threshold for differentiating both clinical diagnoses. Regarding the evaluation of severe fractures using external fixation using the Ilizarov method,
Morasiewicz et $\mathrm{al}^{22}$ conducted research on the possibility of using thermography to monitor bone regeneration during the process of stretching the tibial bone with the use Ilizarov external circular fixator. Due to the increased metabolism expressed in temperature changes, evaluating the thermal changes in the affected limb supports the treatment with additional information to the surgeon. Images were acquired in 18 adult patients and the results showed a significant correlation between bone regeneration (evaluated by radiographic images), compared to thermographic images in the clinical follow-up of patients. The robust correlation between the regenerated and thermal indices measured with the Spearman correlation index allows a reduction in the number of radiographic studies of up to $50 \%$, in most of the cases studied. Mean values of Spearman correlation coefficient for the tibia and femur were $r S=0.925(p<0.01)$ and $0.724(p<0.05)$, respectively.

Haluzan et $\mathrm{al}^{15}$ observed thermal variations of the fractured wrist between 1- and 11-weeks postinjury. At the first week postinjury, the acute inflammatory response is about to finish, and the reparative phase is beginning; angiogenesis and cartilaginous callus formation are taking place, causing an increase in temperature at the fracture site. At week three, the microvascular invasion and perfusion of the fracture site is at its highest, as the cartilaginous callus is vascularized before ossification, causing the greatest increase in temperature. At week five, the callus is beginning to calcify, and the temperature begins to decrease. By week 11, the ossification of the callus continued, and the temperature difference further decrease until the twenty-third week, when the remodeling process will be underway and there is very little difference in temperature between the injured and uninjured wrist.

Given this premise, the present study investigated temperature changes during the healing process in severe tibia fractures, respectively in its three bony portions, proximal, distal and diaphyseal. Comparative analyses were performed with temperature measurements in 20 clinical follow-ups of a male patient during 11 months of treatment in the bone reconstruction department between 2020 and 2021.

The aim of this study was to evaluate the potential of diagnosis and follow-up through infrared thermography when applied to severe orthopedic trauma of the tibia bone, under treatment with an external circular fixator. It is known that thermography can give valuable information on the vascularization, and thus can be helpful to evaluate 
the bone consolidation area as complementary to X-ray images.

\section{Materials and Methods}

The volunteer is a 39-year-old male patient with severe tibial fractures in three places, with bone loss in the distal portion and a gap of $0.14 \mathrm{~m}$. When the patient arrives at the hospital of the clinic, trauma, and bone reconstruction service in Curitiba (PR), Brazil, in 2019, he is diagnosed with pseudarthrosis of the right tibia. In fact, he had been under treatment for five years with a linear fixator at another hospital, without success. Thus, he underwent a therapeutic procedure of osteotomy of the tibia, with a circular external fixator, to initiate bone transport. This surgical approach aims to increase biological support and, consequently, provide greater vascularization for the bone consolidation process.

Thermal images were acquired from June 2020 to May 2021, in 20 patient follow-up visits to assess the clinical evolution, namely with radiographic examinations and for treatment adequacy.

The acquisition of medical thermographic images was performed according to radiographic positioning protocols. A minimum of three sequential images were acquired during the examination, with the patient lying in dorsal decubitus on the clinical table, in anteroposterior view, with the aid of an adjustable support for the thermal imager, as illustrated in Figure 1.

Flir Model T530 Medical Certified Thermal Imager Camera (FLIR ${ }^{\circledR}$ Systems Inc., Wilsonville, Oregon, USA), thermal sensitivity/NETD $<30 \mathrm{mK}$ (at $30^{\circ} \mathrm{C}$ ), lens of $42^{\circ}$ and sensor focal plane array resolution of $320 \times 240$ (76,800 pixels). The equipment was positioned perpendicularly along the axis of the leg, according to the protocol published by Reed et $\mathrm{al}^{17}$ at $0.4 \mathrm{~m}$ from the tibia shaft, allowing visual correlation between thermal and radiological images acquired on the same day. The medical room was maintained with a relative air humidity below $60 \%$, assessed by a portable Mimipa ${ }^{\circledR}$ thermohygrometer, and the ambient temperature, the patient was maintained at a comfort level of $22^{\circ} \mathrm{C}$. Clothes covering the region of diagnostic interest were carefully removed and the patient was invited to attend clinical follow-up visits always wearing loose-fitting clothing that did not squeeze the affected limb, Zhao et al. ${ }^{23}$ The patient was instructed to not have heavy meals, smoke, or drink coffee, alcoholic or energetic stimulants for at least two hours before the appointment. If caffeinated beverages were consumed or antipyretic drugs

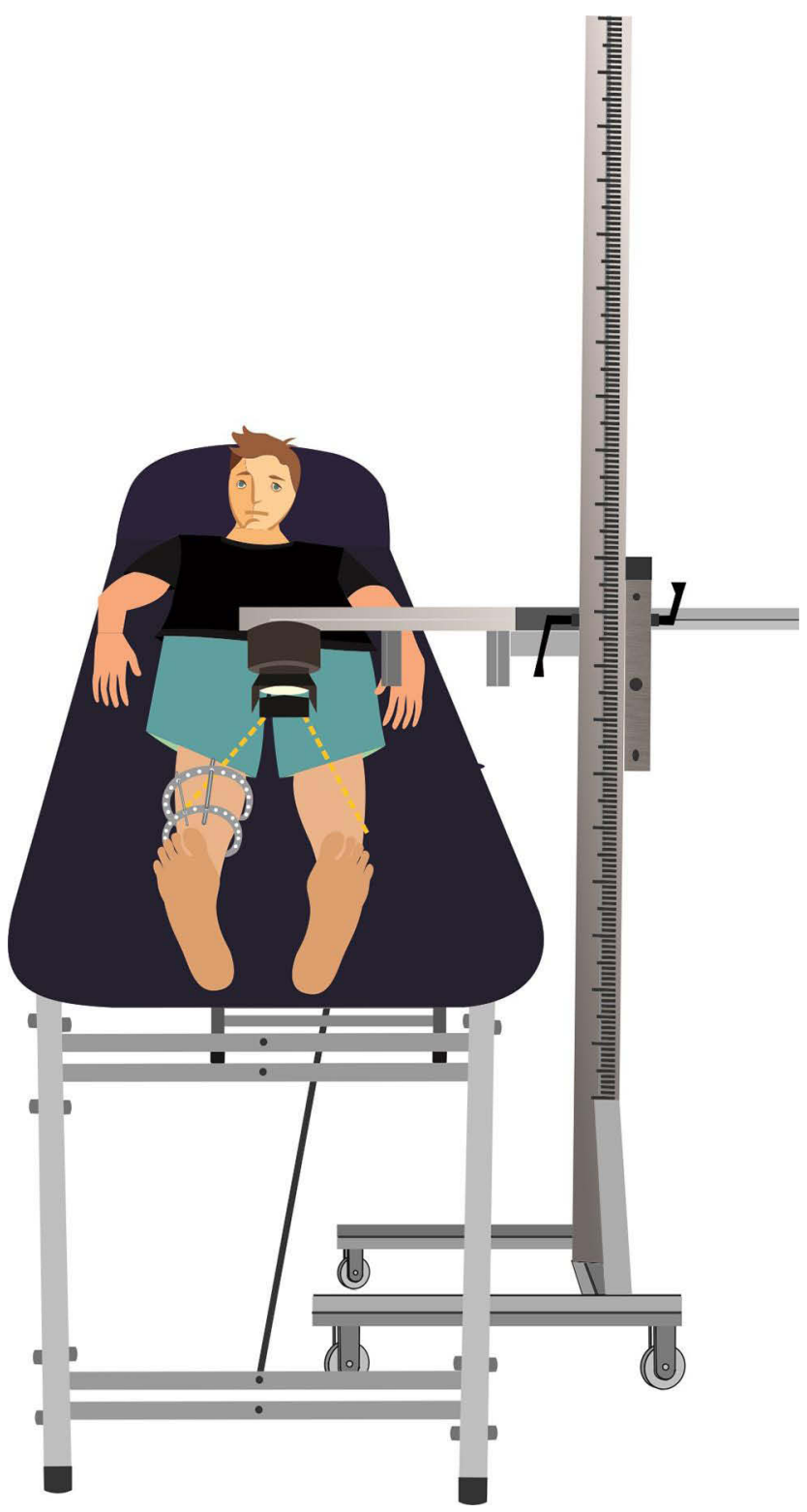

Figure I Illustration schematic of the image acquisition setup.

were administered, the images were not collected during the medical consultation and the patient only underwent radiographic examination. The patient was informed about all the procedures, the importance of the results for public health, and the IRT method.

After the initial clinical conversation with the physician and skin cleansing procedures by specialized nurses, the patient underwent a 15 -min thermal adaptation time to the environment temperature, before thermograms were acquired. Important information such as not crossing legs, not resting hands on thighs were also passed on and supervised by the researcher during the entire time of the patient's thermal acclimatization. The room was 
previously prepared for the acquisition by removing the patient from proximity to metallic medical materials that could cause reflection on the evaluated limb, positioning the clinical stretcher away from electrical wires and energy conducting tubes, minimizing the interference of the infrared energy emanating from external sources. Likewise, only the researcher and the surgeon remain at the time of thermograms acquisition, thus avoiding the increase in temperature in the vicinity of the patient.

This study met the ethical recommendations of Resolution 466/12 and was approved by the Research Ethics Committee of the Federal Technological University of Paraná - UTFPR number 3014748, of November 12, 2018 and by Research Ethics Committee of the Hospital das Clínicas of the Federal University of Paraná HC/UFPR - number 3067005 on December 8, 2018. The patient has signed an informed consent, giving permission to the research team to capture, analyze and publish the personal data and images.

The mean temperatures of the proximal tibia, shaft and distal were analyzed, without including the insertion rings of the ECFIM. To calculate the temperature distribution, the tibia region was first segmented to exclude the background. A binary mask was built in MATLAB ${ }^{\circledR}$ to select the regions of interest to extract the temperature information.

Initially, the thermographic images were visually inspected by the researcher to define the ROIs: proximal and medial portions $(15 \times 50$ pixels $)$ and distal tibia region $(12 \times 30$ pixels $)$, with a rectangular shape both in affected limb, and contralateral, as shown in Figure 2.

The regions were defined considering the central portion of the leg to avoid round areas of the body. Then, a Matlab algorithm was used to crop each ROI, corresponding to a sub-matrix of temperature values. Each matrix was reshaped into a vector, from which the outliers were removed using the rmoutliers function. A value was considered an outlier if farther than threescaled mean absolute deviation (MAD) from the median. The scaled MAD is defined as

Being $X$ the vector of temperatures of a region and $\tilde{X}=\operatorname{median}(X)$. The scalar $c$ is calculated by:

$$
c=\frac{-1}{\sqrt{2} \operatorname{erfcinv}(3 / 2)}
$$

The median of each vector was calculated to reduce the dimensionality of the temperature to a single value for analysis. Data were analyzed using the Wilcoxon signedrank test, for independent samples, comparing the median temperatures along the leg under treatment, with the contralateral leg $(\mathrm{CL})$.

\section{Results}

This chapter presents the infrared and X-ray images token for 11 months, corresponding to 20 follow-ups. In order to make it short, only the most relevant follow-ups are shown in Figures 3-7.

Clinical follow-up 1, by June 18, 2020, (3a) Infrared image and (3b) X-ray, after surgical process of repositioning the EFIM in the right foot. Clinical follow-up 2, July 16, 2020, (3c) infrared image and (3d) X-ray of the surgical procedure and bone transport process in the proximal tibia. Clinical follow-up 3, July 30, 2020, (3e) infrared image and (3f) X-ray to assess the biological response to the bone transport process. In these three initial diagnostic evaluations, the patient was instructed not to rest his foot on the ground, due to the fragility of the affected limb,
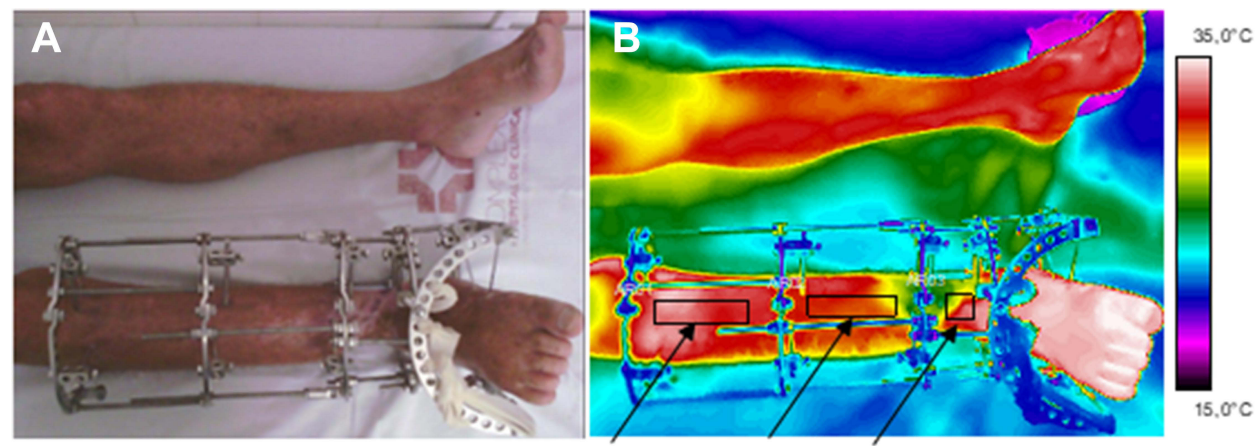

ROI 1 ROI 2 ROI 3

Figure 2 Photograph of the evaluated limb (right leg) (A); Infrared image and defined ROIs (B). ROII -proximal tibia. ROI2 - tibia shaft (diaphyseal), ROI3 - distal tibia. Abbreviation: ROI, regions of interest. 


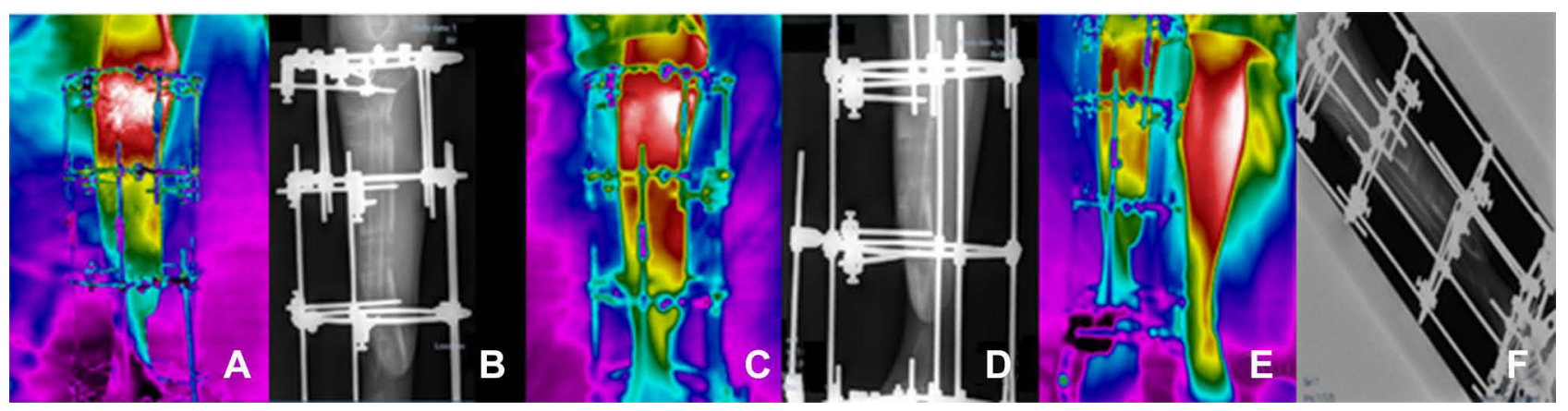

Figure 3 Clinical follow-ups I (A and B), 2 (C and D) and 3 (E and F); (A, C, E) infrared images and (B, D, F) X-ray.

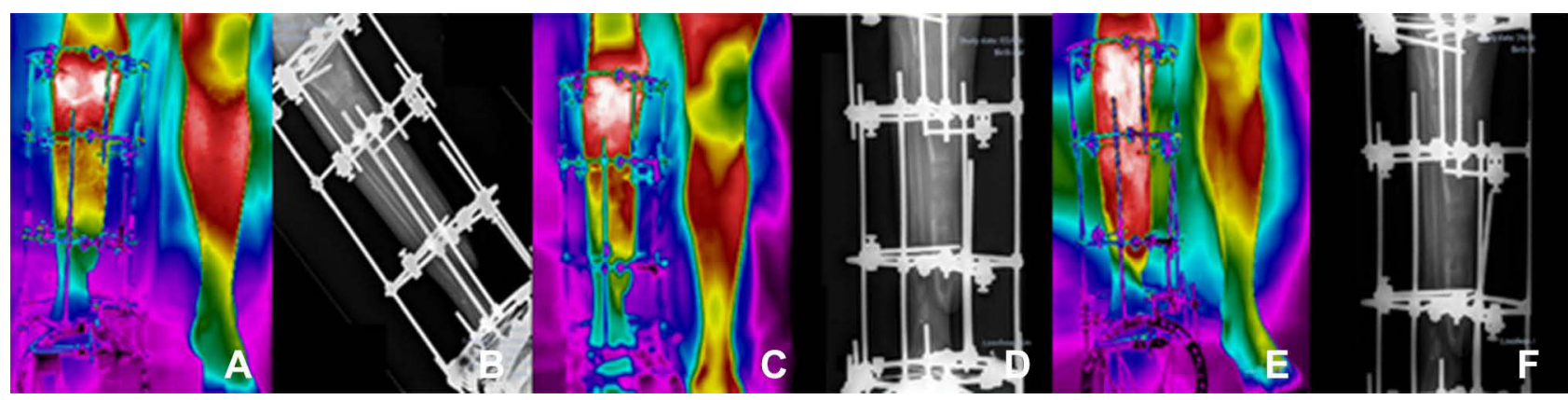

Figure 4 Clinical follow-ups 4 (A and B), 5 (C and $\mathbf{D})$ and 6 (E and F); (A, C, E) infrared images and (B, D, F) X-ray.
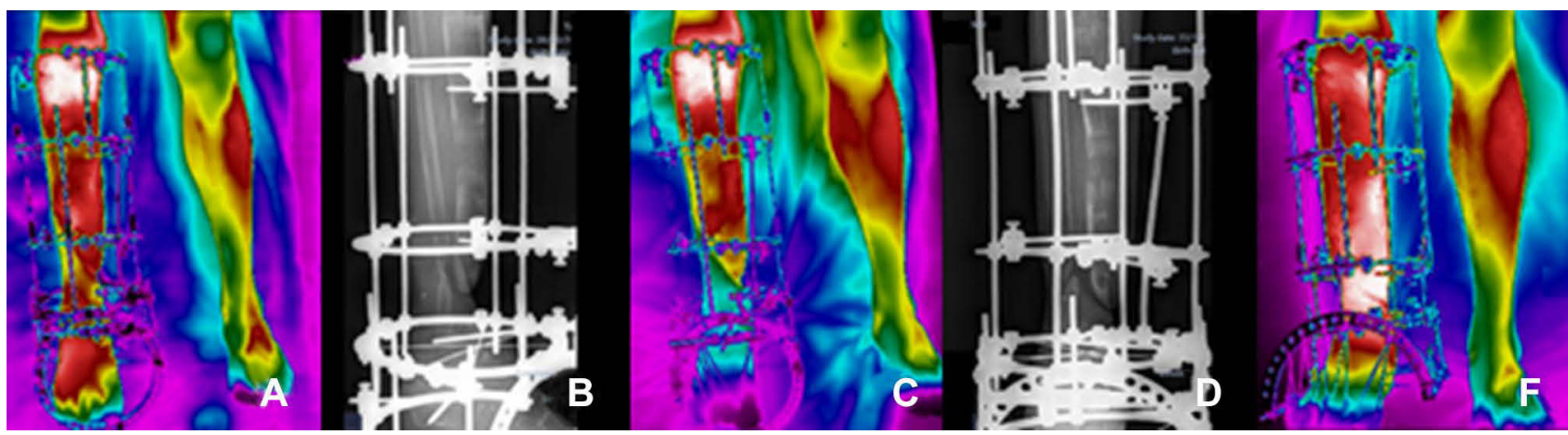

Figure 5 Clinical follow-ups 7 (A and B), 8 (C and D) and 9 (F), (A, C, F) infrared image and (B and D) X-ray.

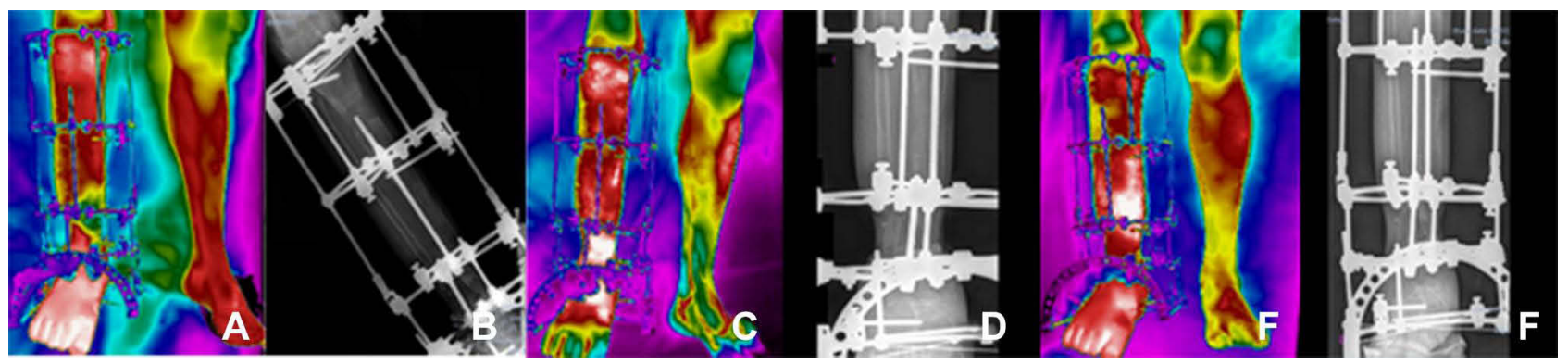

Figure 6 Clinical follow-ups 12 (A and B), 14 (C and D) and 16 (E and F), (A, C, E) infrared image and (B, D, F) X-ray. 


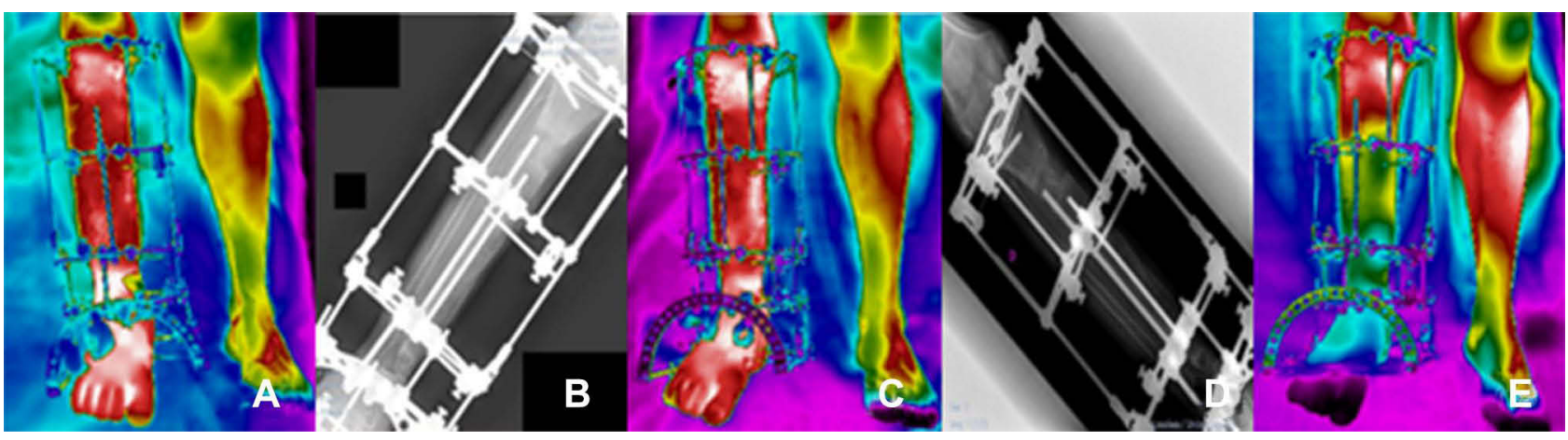

Figure 7 Clinical follow-ups 17 (A and B), 19 (C and D) and 20 (E), (A, C, E) infrared image and (B, D) X-ray.

according to the sequence of images shown in the Figure 3.

Clinical follow-up 4, August 20, 2020, (4a) Infrared image and (4b) X-ray, to perform a new surgical procedure and reassess the ECFIM adjustments. Evaluation of skin infiltration in bone elongation in the distal portion of the tibia. Clinical follow-up 5, September 3, 2020, (4c) infrared image and (4d) radiographic analysis of the evolution of skin infiltration in bone elongation, in the distal portion of the tibia, for a future surgical procedure to remove the skin that presents invagination around the bone. Clinical follow-up 6, September 24, 2020, (4e) infrared image and (4f) X-ray, after adjustment of the external circular fixator. The patient stopped the adjustment for proximal elongation and intensified the adjustment in the distal portion. The bone transport process was started with ECFIM adjustment of a $1 / 4$ turn performed twice a day, according to the sequence of images shown in Figure 4.

Clinical follow-up 7, October 8, 2020, (5a) infrared image and (5b) X-ray, one week after performing a new ECFIM adjustment, the patient presented symptoms of pain in the distal portion of the tibia, due to the tip of the bone pushing the skin. Clinical follow-up 8, October 22, 2020, (5c) infrared image and (5d) X-ray, performed one week after performing a new EFIM adjustment, the patient still had strong painful symptoms in the distal portion of the tibia, due to advancement bone stretching and forcing the skin surface, provided by the increased distension of the skin invagination around the bone. Clinical follow-up 9, November 5, 2020, (5f) infrared image, for clinical follow-up after surgery to remove the invaginated skin, complete bone elongation and removal of the circular portion of the ECFIM below the right foot. Two-month forecast for a new surgery. The limb undergoing treatment is shorter than the contralateral.
Continued with bone lengthening protocol. Prescription to perform stretching in the proximal tibia for another week. Prediction of vascularized fibula surgery for compression and stability of the iliac bone graft already performed. X-ray exam was not performed, according to the sequence of images shown in Figure 5.

Regarding Clinical follow-up 10, on November 19, 2020 , the patient had no diagnostic evolution in the treatment. However, in the next one, follow-up 11, one week after, there was suspicion of edge necrosis in a surgical incision in the distal region of the tibia, therefore, the patient was immediately referred to hospital admission and underwent a new surgery to prevent the infection from progressing to bone regeneration.

Clinical follow-up 12, January 7, 2021, (6a) infrared image and (6b) X-ray, for postoperative evaluation, the patient still has a small scar on the distal portion of the tibia. Amikacin antibiotic prescription to control bone infection secretion. Start of right foot support on the ground to provide biological stimulus for bone consolidation. At the follow-up appointment 13, on January 14, 2021, the medical images of the surgical incision showed good healing status, being fully closed, with no secretion and no symptoms of pain. There was a decrease in edema in the distal tibia and portion of the right foot. Clinical follow-up 14, January 21, 2021, (6c) infrared image and (6d) X-ray, to monitor the healing of the surgical wound, special dressings and laser application on the edges of the incisions were maintained, which showed a skin with small imperfections. Antibiotic prescriptions remained unchanged. Referring to follow-up 15, January 28, 2021, complications in treatment were recurrent, persistent postsurgical infection was observed and, therefore, the patient was instructed to continue the weekly assessment. Clinical follow-up 16, February 4, 2021, (6e) infrared image and 
(6f) X-ray, small surgical wound hole closure was not completed. So far, the patient has continued to present postoperative infection even with administration of potent antibiotics. Special weekly dressing procedures were maintained, according to the sequence of images shown in Figure 6.

Clinical Follow-up 17, February 18, 2021, (7a) infrared image and (7b) X-ray, performed with a special dressing. Recommendation of walking with the support of the limb under treatment. Explication (to the patient) of the contribution of the body weight to stimulate the bone healing process. The piezoelectric action of mechanical load in the diagnosis of bone nonunion provides positive effects on bone metabolism. There was a reduction in foot and ankle edema and, therefore, complementary treatment protocols were maintained through laser therapy and continuity of outpatient procedures of special dressings. In follow-up 18, March 4, 2021, medical images were obtained for clinical follow-up one week after the patient had started the process of supporting both feet on the ground for gait maintenance. In this medical evaluation, the removal of the support crutches was advised to start the process of correct walking, providing an increase in the piezoelectric effect to the bone healing process. In addition to the clinical evaluation, the continuation of treatment protocols with laser therapy in the surgical incision was prescribed, and outpatient procedures for special dressings were maintained. Follow-up 19, March 18, 2021, (7c) infrared image and (7d) X-ray, beginning of release some bars of the external circular fixator to allow a soft adaptation to weight. The patient was advised to walk without crutches. Some corrections were made for adequate foot support to the ground, nonflexion and extension of the knee joint during gait. The clinical guidelines for outpatient dressings remained unchanged. Clinical follow-up 20, May 13, 2021, (7e) Infrared image, for clinical reassessment of the surgical wound treatment protocol with the use of laser therapy and extension of outpatient dressing. Through thermography images, the diagnostic evaluation of decreased peripheral vascular supply at the focal point of bone repair in the distal portion of the tibia was observed, as well as the decrease in temperature throughout the entire portion of the right foot. X-ray exam was not performed, according to the sequence of images shown in Figure 7.

In view of the medical images, important differences were observed of the median temperatures evaluated in the proximal tibia, diaphysis, and distal regions (corresponding to segmental bone loss of $0.14 \mathrm{~m}$ ), along the treatment procedures with the external circular ECFIM. Likewise, the thermal evaluation was helpful in the diagnosis of postsurgical infection. The median temperature was evaluated in the ROIs of the proximal tibia, diaphysis and distal, as shown in Figure 8. During the first phase of the treatment (follow-ups 1 to 10), the diaphysis and distal portions of the tibia are colder, than the proximal, while afterwards the three temperatures become closer. In clinical follow-ups 2 and 3 , the median temperatures measured in the diaphysis of the tibia were $22.4^{\circ} \mathrm{C}$ and $21.1^{\circ} \mathrm{C}$, and in the distal tibia $21.1^{\circ} \mathrm{C}$ and $19.3^{\circ} \mathrm{C}$, respectively, the temperatures are below the thermal threshold of bone healing, showing these portions do not have biological stimulus for healing.

In clinical follow-up 4, there was a thermal improvement of the median temperature of the tibia diaphysis, presenting $24.1^{\circ} \mathrm{C}$, but the distal tibia region was still below the thermal threshold, with a median temperature of $20.8^{\circ} \mathrm{C}$.

In the medical reassessments 5 and 6 , there was an increase of the vascularization observed throughout the diaphysis, followed by a median temperatures augment to $29.2^{\circ} \mathrm{C}$ and $32.4^{\circ} \mathrm{C}$, respectively. The results also showed a higher temperature in the upper portion of the distal tibia, with values above $26.0^{\circ} \mathrm{C}$.

Regarding the follow-up 7 and 8, it was observed in the data of image seven a good vascularization in all evaluated portions of the tibia. However, in the medical consultation 8 , it was observed that the skin infiltration at the bone tip (a) of the distal tibia caused a decrease in blood supply at the focal point of transportation bone. Thus, showing a decrease in the median temperature to $25.4^{\circ} \mathrm{C}$, with an important thermal difference of $4.5{ }^{\circ} \mathrm{C}$, contrasting with the temperature of the previous clinical follow-up of $30.0^{\circ} \mathrm{C}$. In view of this, the thermal image after removal of the skin that entered the bone in the section (a) identified in follow-up 8, showed an improvement in vascularization on the distal tibia portion with a temperature of $33.1^{\circ} \mathrm{C}$, as well as in the entire limb undergoing treatment with median temperature of $31.5^{\circ} \mathrm{C}$, indicating a normalization, in accordance with the normality standards established by the international consensus and guidelines for medical thermography, follow-up 9.

On the clinical follow-up 10, the patient was diagnosed with postsurgical infection, caused by the ECFIM pin on the side of the foot, in the portion of the talus bone, with a decrease in the median temperature in the diaphysis 


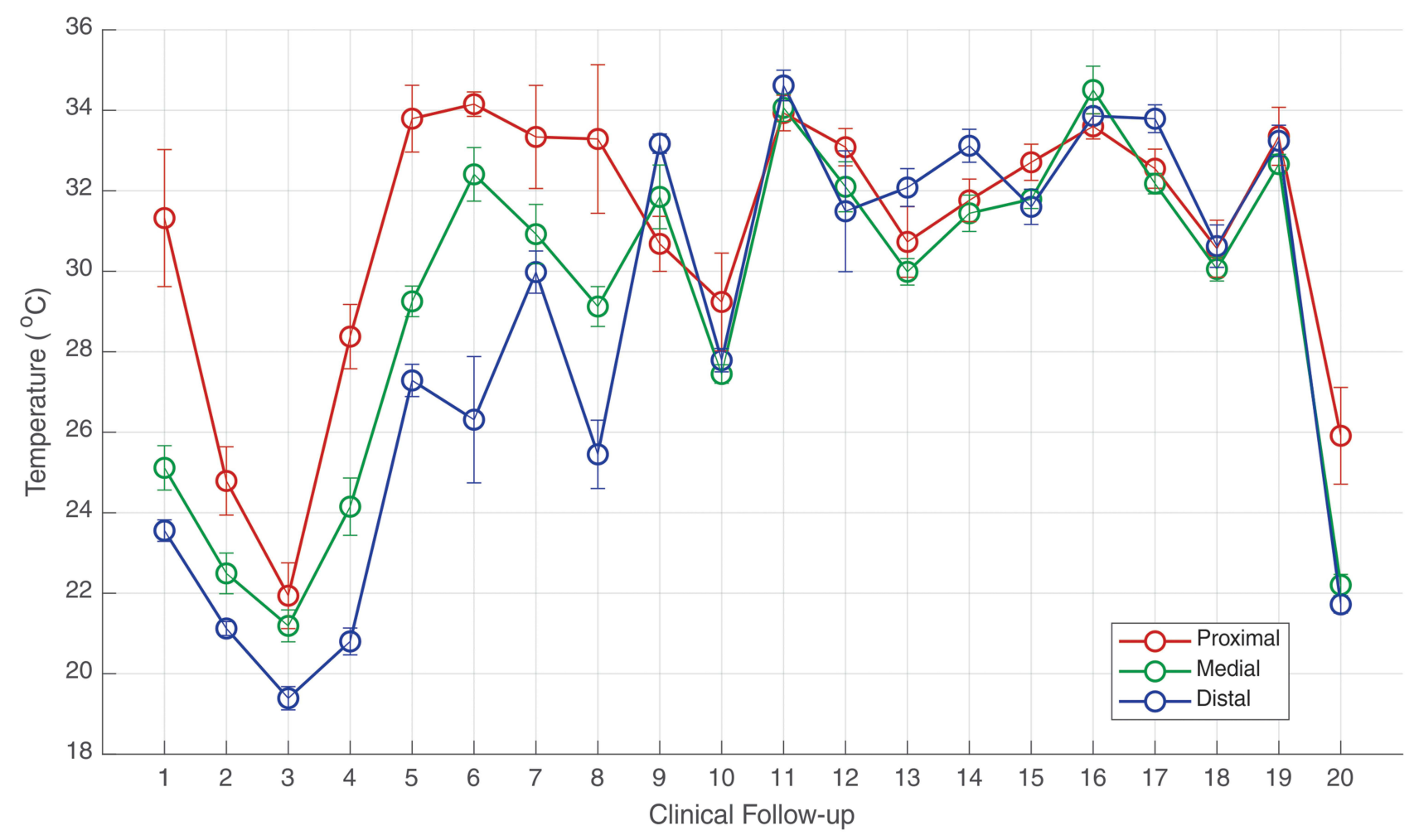

Figure 8 Evolution of median temperature in the ROls of the right leg (under treatment) in the 20 clinical follow-ups. The error bars correspond to one standard deviation.

$\left(27.4^{\circ} \mathrm{C}\right)$ and in the distal tibia portion $\left(27.8^{\circ} \mathrm{C}\right)$, the results of thermographic analysis were in accordance with the results of laboratory tests.

After 15 days, evaluation 11, the patient presented an edema in the ankle and foot region, worsening the diagnosis of postsurgical infection, severe pain in the final portion of the diaphysis and distal tibia. Thus, he was referred to hospital for surgical procedure of debridement and focal cleansing of the bone infection, the clinical examination was confirmed with the analysis of thermal images that demonstrate temperature change in the entire limb under treatment, with greater accentuation in the diaphysis portions of tibia $\left(34^{\circ} \mathrm{C}\right)$ and distal tibia $\left(34.6^{\circ} \mathrm{C}\right)$, values higher than the median temperature of the proximal tibia portion $\left(33.9^{\circ} \mathrm{C}\right)$. The average temperature of healthy people is $\left(31.5^{\circ} \mathrm{C}\right)$ in the tibia diaphysis, decreasing to the extremities, therefore the temperatures referred to above are abnormally high corroborating the analysis of the infection.

In the subsequent evaluation, 12, a reduction in temperature was observed with thermal distribution within the normal range in all investigated portions.

In clinical segments 13 and 14, there was a progressive healing and absence of infectious foci in the portions of the proximal tibia and tibia diaphysis, but an increase in temperature was again observed in the portion of the distal tibia during this period $\left(32.0^{\circ} \mathrm{C}\right.$ and $33.1^{\circ} \mathrm{C}$, respectively), with suspicion of new infection of the ankle insertion pin. The scar wound still has a tiny orifice, and special dressings and laser treatment were maintained.

Regarding clinical follow-up 15, the patient had a persistent diagnosis of infection, denoting a temperature greater than $31^{\circ} \mathrm{C}$ in all portions of the limb under treatment by means of the ECFIM. After a week of trying to solve the infections, the median temperature once again increased, affecting the entire limb, in particular the tibia diaphysis $\left(34.5^{\circ} \mathrm{C}\right)$, showing no improvement in the diagnosis.

Regarding the clinical follow-up 17, it was observed in the distal tibia and foot portion that the temperature is compatible with the foot support on the ground, the thermal values still too high $\left(33.8^{\circ} \mathrm{C}\right)$, the region was very sensitive, manifesting painful symptoms due to body weight load at the focal point of the distal bone regenerated. This plantar support is recommended to provide mechanical stimulation to bone healing. The temperature denoted little improvement with thermal decrease in the proximal tibia to $32.5^{\circ} \mathrm{C}$ and in the diaphysis to $32.1^{\circ} \mathrm{C}$. 
Regarding the thermal evaluation of follow-up 18, there was stabilization of bone healing without significant changes in thermal magnitude, the median temperature remained at $\left(30.0^{\circ} \mathrm{C}\right)$. However, after 14 days of return for a new reassessment of the healing process, it was observed a change in the mean temperature with higher value in the distal tibia portion with values equal to the proximal tibia $\left(33.2^{\circ} \mathrm{C}\right)$. These data characterize the possibility of a reminiscence infectious process in the distal portion.

In the last clinical follow-up performed using medical thermography (20), there was a drastic reduction in median temperature in the entire limb under treatment, as the interval between the previous clinical follow-up appointment was 57 days, since COVID-19 pandemic priority forced postponement of the scheduled appointments. The results of the thermal evaluation demonstrated that the bone graft in the distal region stopped providing blood supply to the bone regenerate and thus the healing process became reduced, especially in the tibia shaft portions with a median temperature of $22.2^{\circ} \mathrm{C}$ and the distal tibia $21.7^{\circ} \mathrm{C}$.

In accordance with the statistical analysis performed by the Wilcoxon rank sum test for independent samples for the defined ROIs, the data showed significant differences in all comparisons performed, between the right leg (under treatment) and the contralateral leg (CL), $p<0.05$.

For a better understanding of the evolution of clinical treatment, in the different clinical procedures and in the appropriate phases of bone healing with the use of the ECFIM, Figure 9 shows the median temperature of both legs during the treatment.

For a more detailed analysis of the assessed proximal tibia, comparisons of the median value of the ROIs were made between the right leg treated with ECFIM and the contralateral, as shown in boxplot displayed in Figure 10.

Detailed median temperatures of the evaluated ROIs of tibia diaphysis, and the contralateral leg (CL) are shown in the boxplot of Figure 11.

In the same way, the distal tibia median temperatures of the evaluated ROIs are shown along with the median CL, boxplot of Figure 12. The right distal tibia (the most severe affected region) presented a bone loss of $0.14 \mathrm{~m}$ at the beginning of the treatment.

\section{Discussion}

The postfracture bone healing process phases are quite different, the first stage is the inflammatory process, where

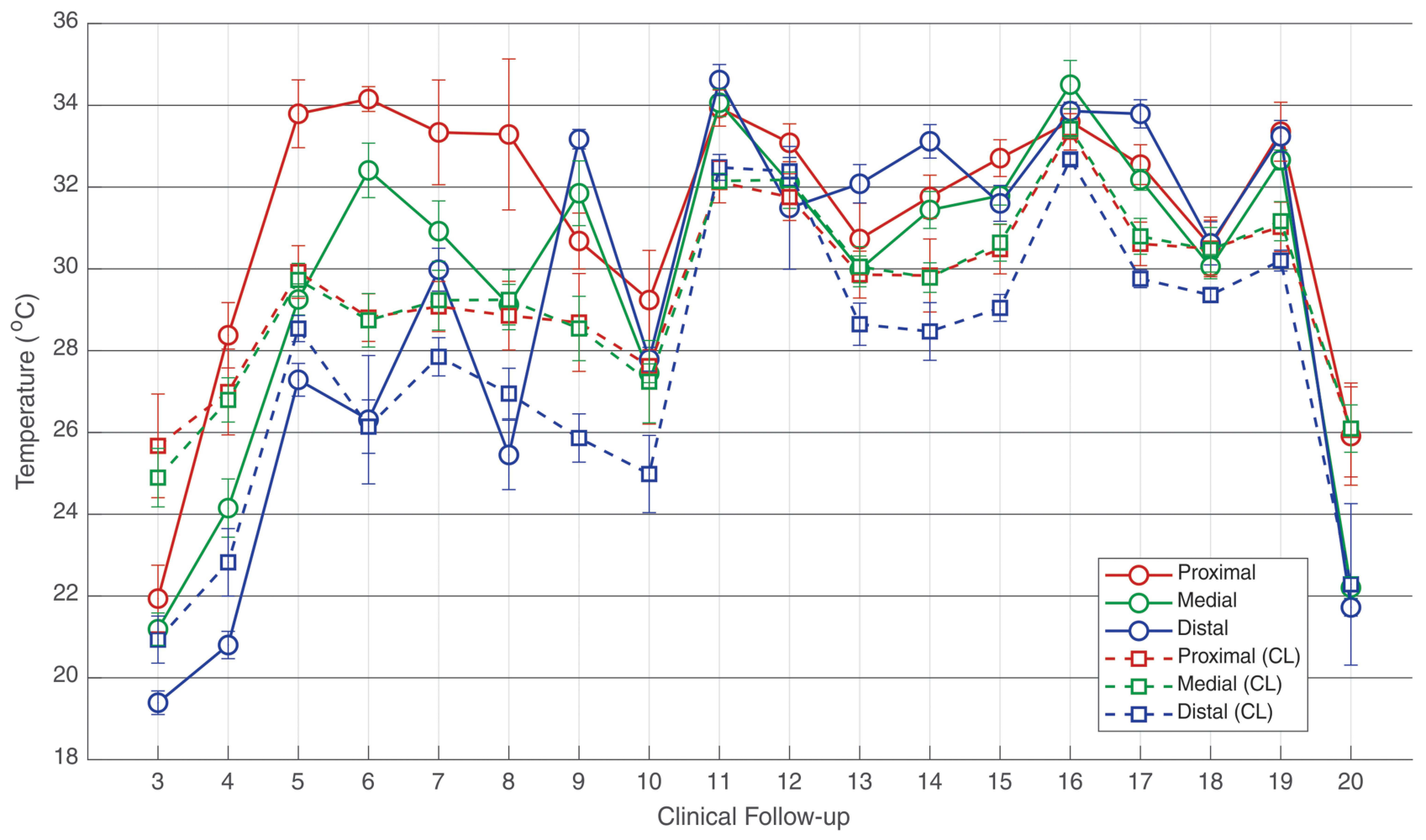

Figure 9 Evolution of median temperature in the ROls of the right leg (under treatment) in the 20 clinical segments using the ECFIM in comparison with the ROls of the healthy left leg $(\mathrm{CL})$. The error bars correspond to one standard deviation. 


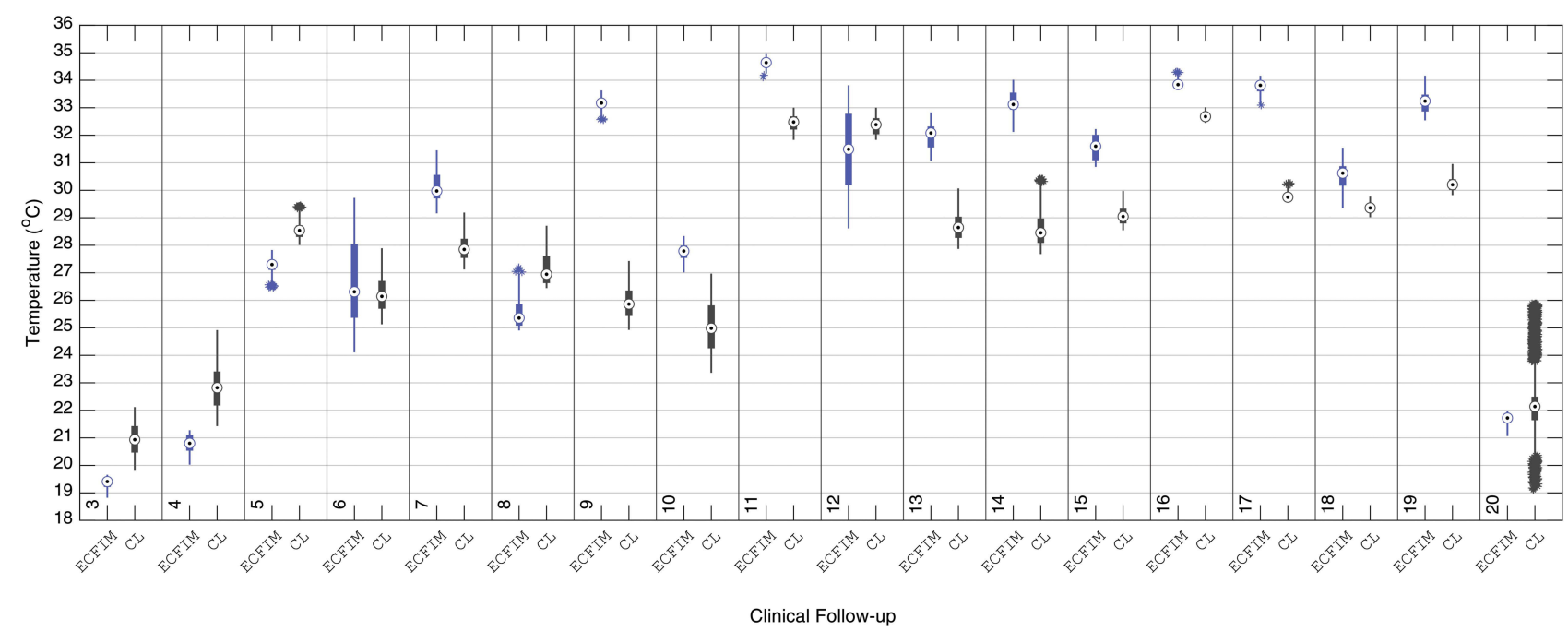

Figure 10 Boxplot of the median temperature of the ROI of the right proximal tibia with the use of the ECFIM, in the 20 follow-up sessions, compared with the ROI of the same portion of the healthy left leg $(\mathrm{CL})$.

Abbreviations: ECFIM, external circular fixator llizarov method; CL, contralateral.

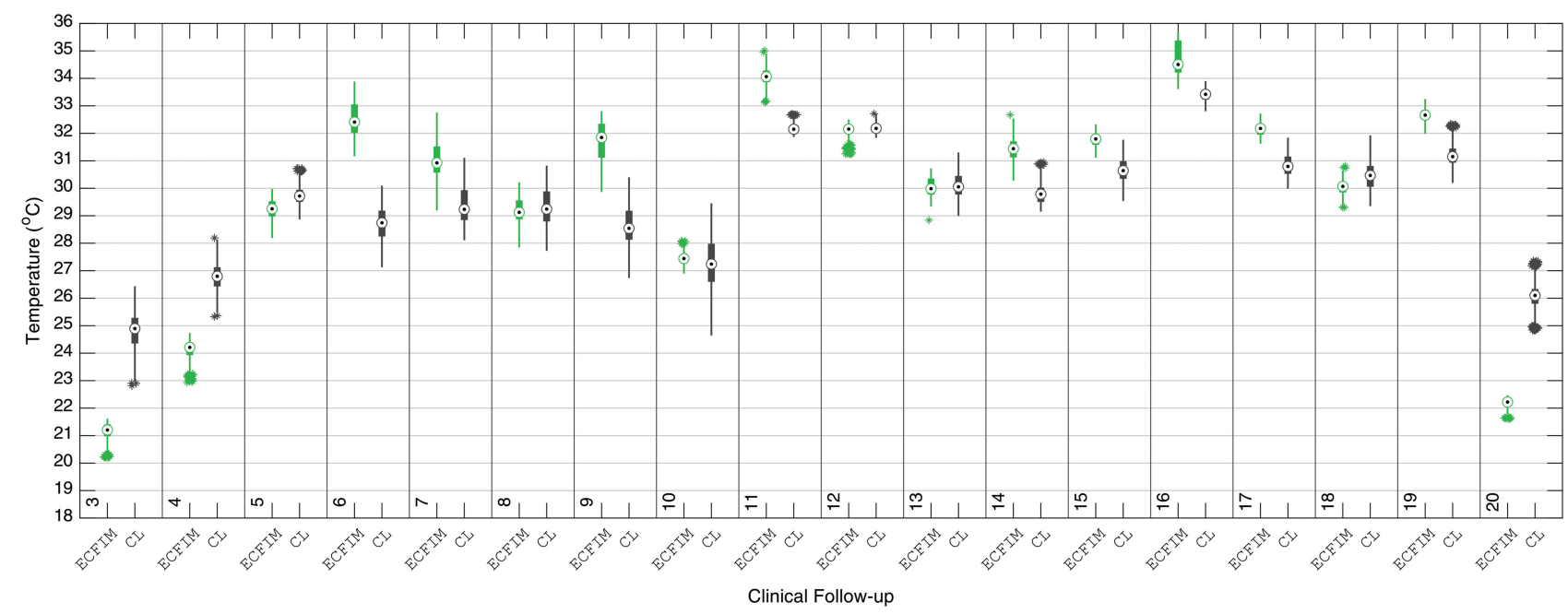

Figure I I Boxplot of the median temperature of the ROI of the diaphysis portion of the right tibia using the ECFIM, in the 20 follow-up sessions, compared with the healthy left leg (CL).

there is an increase in microcirculation at the fracture site, with focal edema and warmer skin areas. The second phase begins bone repair with the formation of soft callus, an increase in internal bone temperature, as well as, in adjacent areas due to the necessary vascular supply to start bone remodeling. ${ }^{22}$ In stage three, where the hard bone callus appears, the temperature begins to decrease, returning only to normal physiological pattern when complete bone remodeling is achieved, which corresponds to phase four of bone healing, according to the thermal amplitude relevant to the body region evaluated. ${ }^{24-26}$
The medical imaging follow-up of the clinical evolution of healing of bone fractures is made through X-ray images, considered the gold standard in the evaluation of orthopedic trauma. ${ }^{27}$ Among medical diagnostic images, digital infrared thermography imaging is a noninvasive and noncontact diagnostic technique, which quantifies the temperature by measuring the infrared radiation emitted by the body surface. ${ }^{26}$

Regarding the evaluation of healthy volunteers who do not present bone fractures, according to the international consensus and guidelines for medical thermography, ${ }^{19}$ the 


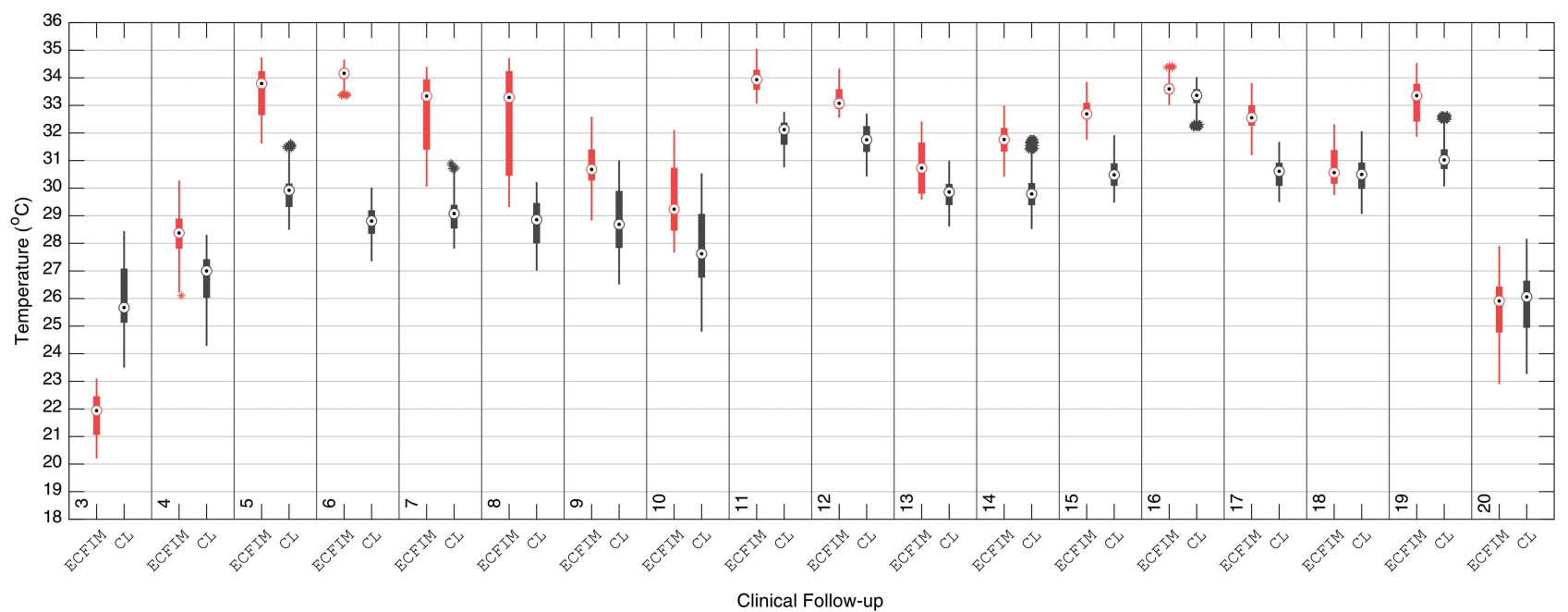

Figure 12 Boxplot of the median temperature of the ROI of the right distal tibia portion (with ECFIM), in the following 20 clinicians, with the ROI of the same portion of the healthy left leg.

median temperature in the leg region corresponding to the tibia bone is $31.5^{\circ} \mathrm{C}$. These data are also supported by the researchers, ${ }^{22}$ who evaluated 175 healthy volunteers (reference group) compared to thermal measurements in patients corresponding to treatment of bone transporter with ECFIM. Clinical follow-ups were carried out through thermographic images and correlated with medical radiographic images in fortnightly periods of return to the medical clinic, in the assessment of new bone healing. The results evidenced metabolic thermal alterations, demonstrating an increase in the leg under treatment with ECEFIM, associated with the bone formation and later its total remodeling. For the right leg, the median temperature was $30.3^{\circ} \mathrm{C}$ and for the left leg $30.5^{\circ} \mathrm{C}$, showing values close to the ones indicated by the international reference standards. ${ }^{19}$

In the diagnosis of nonunion bone, temperatures are colder, due to the absence of sufficient vascular supply for bone remodeling and, therefore, the bone interrupts the healing process. As observed in the temperatures obtained in the two initial evaluations of the investigated patient with very cold median temperature in the right leg treated with ECFIM, with thermal values in the distal tibia of $23.5^{\circ} \mathrm{C}$ (assessment 1 ), and $21.1^{\circ} \mathrm{C}$ in the clinical followup (assessment 2).

In medical thermographic diagnostic analysis, thermal symmetry emerges as a clinical relevant parameter. ${ }^{17,28,29}$ The comparison of thermal images to contralateral limbs enhanced the asymmetry regions which are indicative of pathologies or injuries, as well as changes in local blood flow. ${ }^{28}$ Thermal differences above $0.3^{\circ} \mathrm{C}$ indicate vascular abnormality, values of $0.6^{\circ} \mathrm{C}$ denote an important abnormality that advise a more accurate clinical investigation with other medical imaging tests in the correlation of diagnoses. Nevertheless, differences above $1.0^{\circ} \mathrm{C}$ are a strong indication of abnormality diagnostic. ${ }^{19}$ In view of this statement, and in accordance with the studies by the aforementioned authors, the results of the 11 months of clinical follow-up showed identical thermal behavior. Comparative values of the patient in the right leg under treatment with ECFIM and in the healthy left leg (contralateral limb), in the three ROIs measured in the proximal tibia, tibia diaphysis and distal tibia, the clinical follow-up 3, 4, 5, 7, 9, 11, 14, 15, 17, and 19 showed differences in the median temperature higher than $1{ }^{\circ} \mathrm{C}$ in all analyzed portions, confirming severe thermal abnormality in the lower limb under treatment protocol, with significant thermal difference values. However, in the analysis of data from follow-up 6, the median temperature in the ROI of the distal tibia showed a thermal difference of $0.17^{\circ} \mathrm{C}$, which is significant for normality in the thermal comparison, demonstrating a good vascular response to the stretching process in this region. In clinical follow-ups $8,10,12$, and 13 , when analyzing the tibia shaft, it shows variations among the normal values of body thermal asymmetry, with no median temperature in this portion measured greater than $0.2^{\circ} \mathrm{C}$. The findings of this long clinical follow-up by medical thermography are in agreement with and strongly corroborate the data from the patient's medical record of good clinical evolution of healing in this bone portion. ${ }^{22}$ 
Likewise, in the comparative analysis of the median temperature in the proximal tibia portion, clinical followups 16,18 , and 20 confirm good vascular distribution of the transporting bone performed in this portion, with temperature values within the normal range of thermal symmetry, maintaining the metabolic contribution to the complete cellular action of osteoblasts in the bone remodeling process. These data indicate the medical infrared thermography (IRT) is suitable for monitoring bone healing in severe bone pathologies such as tibia nonunion and other fractures in monitoring the phases of bone remodeling, and suggest agreement with the authors who equally evaluated medical thermography in the assessment of the regenerated bone. $^{21,30}$ This is also supported by Morasiewicz et al who used medical thermography to monitor bone regeneration during lengthening process in 18 patients who presented segmental bone loss in the tibial bone and use ECFIM, with distraction osteogenesis of one millimeter per day. ${ }^{22}$

The results of this important study demonstrated a robust correlation between the bone regeneration and the thermal data obtained, allowing the authors to point out the thermographic examination contributed to a $50 \%$ reduction in radiographic imaging exams in clinical follow-up. Likewise, it was possible to assess the efficacy of medical thermography imaging in the early detection of soft tissue inflammation around the ECFIM Kirschner wires.

The results of Morasiewicz et $\mathrm{al}^{22}$ corroborate the findings of this investigation, where during the 11 months of clinical follow-up, the median of the ROI temperatures evaluated from infrared images in the different phases of treatment with ECFIM are compared to the contralateral limb, and clinical findings. The conclusions proved to be assertive in the correlations with $\mathrm{X}$-ray images, confirming the diagnoses of interruption of the bone regeneration and the verification of infectious processes. The results found by these authors confirm the data obtained from this investigation in thermal imaging evaluations of the interruption of the healing process detected in thermal clinical follow-ups 1, 2, 3, and 20. Thermal images showed very cold areas confirmed by the data processing of ROIs, showing median temperatures in the thermal range of $21^{\circ} \mathrm{C}$ to $22^{\circ} \mathrm{C}$. These thermal values confirm the diagnosis of interruption of bone remodeling according to Haluzan et al and Jaña et al. ${ }^{15,26}$
Referring to bone infection that arouses inflammation in the rigid outer bone layer, with the subsequent advancement to the inner parts of the bone under treatment, the diagnosis of osteomyelitis, ${ }^{31}$ which provokes compression of blood vessels, reducing local microcirculation; consequently, it prevents the healing and leads to bone tissue death. ${ }^{12}$

In view of this, the clinical data evaluated in the infectious processes of the patient under medical followup, had shown correlation with relevant thermal changes measured through thermography, in which the infectious processes had presented a median temperature in the diaphysis of the tibia $\left(35.1^{\circ} \mathrm{C}\right)$ and distal tibia $\left(34.2^{\circ} \mathrm{C}\right)$, values higher than the temperature of the proximal tibia portion $\left(33.9^{\circ} \mathrm{C}\right) .{ }^{27}$

The detected thermal changes allowed additional clinical data to be correlated with the patient radiographic images ${ }^{18}$ which showed the focal points of temperature rise, delimiting treatment areas and clinical decisions for surgical interventions such as bone debridement, the chosen option to prevent the advancement of bone infection and to promote the regeneration of the distal portion of the tibia.

Regarding the number of requests for radiographic examinations to assess the bone healing process and response to bone traction, lengthening procedures and transporting bone treatment, it was observed that in the 20 follow-ups performed, 15 radiological images were requested in lateral and anteroposterior views. The results of the correlation analysis of radiographic and thermographic medical images suggest the possibility that imaging exams in bone trauma can be interspersed, minimizing excessive patient exposure to successive exams with ionizing radiation. According to Morasiewicz et $\mathrm{al}^{22}$ about the same type of bone pathology analyzed herein, it was possible to reduce the number of X-ray exams as well. Thermography can be used in conjunction with traditional diagnostic methods in monitoring bone formation at all stages of limb lengthening. The authors performed analysis of the established thermal index values, based on 72 thermograms performed in the bone regeneration process, during the evaluation of the different healing stages of the bone elongation process. Thermal data were compared with assessment scores from 72 $\mathrm{X}$-ray examinations. The analysis demonstrated statistical significance with a stronger index presented in the evaluation of medical images in the anteroposterior view. The authors also suggest that the examination through medical 
thermography does not replace the performance of radiographic imaging exams, however the thermographic diagnostic technique aims to be an adjuvant complementary exam in the medical prognosis and in cases of regenerated bone formation disorders.

Likewise, in the treatment of bone fractures using ECFIM, when the foot is full contacting the ground, the data obtained by thermal imaging shows a greater local vascularization. This action of increased vascularization is due to mechanical loading stimuli provided to the bone, explained by Wolff's law of mechanotransduction. ${ }^{32}$

The piezoelectric charge generated by the load of body weight exerted on the affected limb, favors the cellular action of osteoblasts at the fracture site. These data were similarly evidenced in our study, in the diagnosis through thermography, according to data obtained in the 20 thermal imaging exams.

The measured temperatures indicated the median values of $32^{\circ} \mathrm{C}$ in the foot region, allowing the physician to confirm that the patient is placing the foot on the ground to support the gait, promoting biological stimulation of the bone healing. IRT adds important physiological information of the bone healing process, being therefore relevant for the clinicians. ${ }^{33}$ In this context, thermographic analysis may indicate changes and/or disturbances in the bone repair process, presenting itself as a complementary diagnostic method to minimize the subjectivity of medical reports, corroborating with other relevant additional information not included in diagnoses made by conventional methods of imaging. The temperature map of the affected limb can support the prescription of additional immobilization time, individualized rehabilitation protocol, as well as new surgery.

The analysis of the thermograms support the evaluation of the healing process, where cooler periods denote interruption of the bone reparation process while local infection and vascularization increase the temperature, enabling a more precise clinical approach, by complementing the information of standard medical images. IRT allows a reduction of invasive exams and, therefore, the ionizing radiation in the clinical follow-up visits by interspersing them with thermographic exams.

\section{Conclusion}

Medical infrared thermography is a safe image technique that can be successfully applied to orthopedic trauma with bone segmental loss and the use of an external circular fixator, as in this case study, and the data is correlated with the gold standard exams, X-ray, for evaluating the bone healing process.

Clinical follow-up by thermography in the different stages of bone remodeling, including fixator adjustment steps for mechanical action of traction or bone lengthening, as well as infectious and postsurgical bone graft processes, appear to provide additional physiological data of vascularization of the bone in regenerative process. IRT provides the physician with additional information for the clinical follow-up of the patient in the adjustments of the treatment protocols, means for reducing the number of $\mathrm{X}$-ray exams and, consequently, to the ionizing radiation.

\section{Acknowledgments}

This research was founded by Araucária Foundation and $\mathrm{CNPq}$ for scholarships and support for the Coordination for Improvement of Higher Education Personnel - Brazil (CAPES) - Financing Code 001, and Project LAETAUIDB/50022/2020, UIDP/50022/2020.

The abstract of a very preliminary version of this paper was presented at the QIRT 2020 Conference as an oral talk archived in online proceedings http://qirt.org/archives/ qirt2020/papers/155.pdf.

\section{Disclosure}

The authors report no conflicts of interest in this work.

\section{References}

1. Oliveira O, Martins SPR, Lima W, Gomes MM. The use of bone morphogenetic proteins (BMP) and pseudarthrosis, a literature review. Revista Brasileira De Ortopedia. 2017;52:124-140. doi:10.1016/j.rboe.2016.03.005

2. Ulson O, Zamboni C, Durigan JR, et al. Treatment of femur pseudoarthrosis using wave plate: evaluation of consolidation and its relationship with graft type. Injury. 2021;52:S18-S22. doi:10.1016/j. injury.2021.01.045

3. Ilizarov G, Deviatov A, Trokhova V. Surgical lengthening of the shortened lower extremities. Vestnik Khirurgii Imeni II Grekova. 1972;108(2):100-103.

4. Shortt NL, Keenan GF, Muir AY, Simpson A. The use of a nerve stimulator to allow safe placement of Ilizarov wires. Oper Orthop Traumatol. 2006;18(4):364-376. doi:10.1007/s00064-006-1177-2

5. Peng J, Min L, Xiang Z, Huang F, Tu C, Zhang H. Ilizarov bone transport combined with antibiotic cement spacer for infected tibial nonunion. Int J Clin Exp Med. 2015;8(6):10058. doi:10.3390/ ijerph 18084217

6. Eralp IL, Kocaoğlu M, Dikmen G, Azam ME, Balcı Hİ, Bilen FE. Treatment of infected nonunion of the juxta-articular region of the distal tibia. Acta Orthop Traumatol Turc. 2016;50(2):139-146. doi:10.3944/AOTT.2015.15.0147

7. Hasankhani E, Payvandi MT, Birjandinejad A. The Ilizarov ring external fixator in complex open fractures of the tibia. Eur J Trauma. 2006;32(1):63-68. doi:10.1007/s00068-005-0031-6 
8. Rohilla R, Wadhwani J, Devgan A, Singh R, Khanna M. Prospective randomised comparison of ring versus rail fixator in infected gap nonunion of tibia treated with distraction osteogenesis. Bone Joint J. 2016;98(10):1399-1405. doi:10.1302/0301-620X.98B10.37946

9. Szelerski Ł, Kozłowska AP, Żarek S, et al. A new criterion for assessing Ilizarov treatment outcomes in nonunion of the tibia. Arch Orthop Trauma Surg. 2021;141(5):879-889. doi:10.1007/s00402-020-03571-8

10. Kristiansen LP, Steen H. Reduced lengthening index by use of bifocal osteotomy in the tibia: comparison of monofocal and bifocal procedures with the Ilizarov external fixator. Acta Orthop Scand. 2002;73 (1):93-97. doi:10.1080/000164702317281486

11. Deng Z, Mu Y, Sun X, Xu Y, Li F, Yin L. A systematic review and meta-analysis of combined antibiotic spacer with Ilizarov methods in the treatment of infected nonunion of tibia. Biomed Res Int. 2021;2021:1-10. doi:10.1155/2021/6668617

12. Sharma S, Kumar A, Sinha S. Role of Ilizarov ring fixator-A three parameter test. Int J Health Clin Res. 2021;4(5):26-29.

13. Casanova MR, Oliveira C, Fernandes EM, et al. Spatial immobilization of autologous growth factors to control vascularization in bone tissue engineering. Biomater Sci. 2019;8. doi:10.1039/d0bm00087f

14. Claes L, Shefelbine S, Schütz M, Willie B. Improvement of clinical fracture healing-what can be learned from mechano-biological research? J Biomech. 2020;110148. doi:10.1016/j.jbiomech.2020.110148

15. Haluzan D, Davila S, Antabak A, et al. Thermal changes during healing of distal radius fractures-preliminary findings. Injury. 2015;46:S103-S106. doi:10.1016/j.injury.2015.10.046

16. Selvarasu N, Nachiappan A, Nandhitha N. Abnormality detection from medical thermographs in human using Euclidean distance based color image segmentation. In 2010 International Conference on Signal Acquisition and Processing. IEEE; 2010: 73-75.

17. Reed C, Saatchi R, Burke D, Ramlakhan S. Infrared thermal imaging as a screening tool for paediatric wrist fractures. Medical Biol Eng Comput. 2020;58(7):1549-1563. doi:10.1007/s11517-020-02167-z

18. Auf der Strasse W, Prado Campos D, Aguiar Mendonça CJ, Mendes J, Soni JF, Nohama P. Thermal variations in osteoporosis after aclasta $^{\circledR}$ administration: case study. Int J Online Biomed Engi. 2020;16(10). doi:10.3991/ijoe.v16i10.14635

19. Ring EFJ, Ammer K. Infrared thermal imaging in medicine. Physiol Meas. 2012;33(3):R33-R46. doi:10.1088/0967-3334/33/3/r33

20. $\mathrm{Hu} \mathrm{K}$, Olsen BR. The roles of vascular endothelial growth factor in bone repair and regeneration. Bone. 2016;91:30-38. doi:10.1016/j. bone.2016.06.013

21. De Salis AF, Saatchi R, Dimitri P. Evaluation of high resolution thermal imaging to determine the effect of vertebral fractures on associated skin surface temperature in children with osteogenesis imperfecta. Med Biol Eng Comput. 2018;56(9):1633-1643. doi:10.1007/s11517-018-1806-3
22. Morasiewicz L, Dudek K, Orzechowski W, Kulej M, Stepniewski M. Use of thermography to monitor the bone regenerate during limb lengthening-preliminary communication. Ortop Traumatol Rehabil. 2008;10(3):279-285.

23. Zhao Y, Iyer RS, Reichley L, et al. A pilot study of infrared thermal imaging to detect active bone lesions in children with chronic nonbacterial osteomyelitis. Arth Care Res. 2019;71(11):1430-1435. doi:10.1002/acr.23804

24. Maruyama M, Rhee C, Utsunomiya T, et al. Modulation of the inflammatory response and bone healing. Front Endocrinol (Lausanne). 2020;11:386. doi:10.3389/fendo.2020.00386

25. Devitt J. Musculoskeletal Healing Process. Sports-Related Fractures, Dislocations and Trauma. New York, NY: Springer International Publishing; 2020. 97-104. doi:10.1007/978-3-030-36790-9 10

26. Jaña FC, Canal M, Alves BAF, Ferreira PM, Ayres JC, Alves R. Analysis of the characteristics of patients with open tibial fractures of

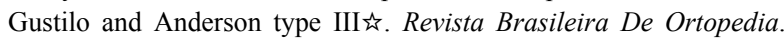
2016;51(2):143-149. doi:10.1016/j.rboe.2016.01.002

27. Litrenta J, Tornetta III P, Mehta S, et al. Determination of radiographic healing: an assessment of consistency using RUST and modified RUST in metadiaphyseal fractures. J Orthop Trauma. 2015;29 (11):516-520. doi:10.1097/BOT.0000000000000390

28. Hildebrandt C, Zeilberger K, Ring EFJ, Raschner C. The Application of Medical Infrared Thermography in Sports Medicine. INTECH Open Access Publisher; 2012.

29. Clemente MP, Mendes J, Vardasca R, et al. Infrared thermography of the crânio-cervico-mandibular complex in wind and string instrumentalists. Int Arch Occup Environ Health. 2020;93(5):645-658. doi:10.1007/s00420-020-01517-6

30. Blasco JM, Sanchis-Sánchez E, Martín JD, Sanchis E, SalvadorPalmer R, Cibrián R. A Matlab based interface for infrared thermographic diagnosis of pediatric musculoskeletal injuries. Infrared Phys Technol. 2016;76:500-503. doi:10.1016/j.infrared.2016.04.018

31. Testa G, Vescio A, Aloj DC, et al. Treatment of infected tibial non-unions with Ilizarov technique: a case series. J Clin Med. 2020;9(5):1352. doi:10.3390/jcm9051352

32. Assanah F, Khan Y. Cell responses to physical forces, and how they inform the design of tissue-engineered constructs for bone repair: a review. J Mater Sci. 2018;53(8):5618-5640. doi:10.1007/s10853-017-1948-y

33. Sanchis-Sánchez E, Vergara-Hernández C, Cibrián RM, Salvador R, Sanchis E, Codoñer-Franch P. Infrared thermal imaging in the diagnosis of musculoskeletal injuries: a systematic review and meta-analysis. $\mathrm{Am}$ J Roentgenol. 2014;203(4):875-882. doi:10.2214/AJR.13.11716
Journal of Multidisciplinary Healthcare

\section{Publish your work in this journal}

The Journal of Multidisciplinary Healthcare is an international, peerreviewed open-access journal that aims to represent and publish research in healthcare areas delivered by practitioners of different disciplines. This includes studies and reviews conducted by multidisciplinary teams as well as research which evaluates the results or conduct of such teams or healthcare processes in general. The journal

Submit your manuscript here: https://www.dovepress.com/journal-of-inflammation-research-journal covers a very wide range of areas and welcomes submissions from practitioners at all levels, from all over the world. The manuscript management system is completely online and includes a very quick and fair peer-review system. Visit http://www.dovepress.com/testimonials. php to read real quotes from published authors. 